\title{
Será a dislexia uma vantagem? Um olhar diferente sobre a diferença
}

\author{
Catarina Mangas ${ }^{1}$ \\ ${ }^{1}$ ESECS, CICS.NOVA.IPLeiria - iACT, CI\&DEI, Politécnico de Leiria, Portugal |
} catarina.mangas@ipleiria.pt | https://orcid.org/0000-0003-0843-5861

Resumo: A dislexia é uma Dificuldade de Aprendizagem Específica associada, essencialmente, a fragilidades ao nível da competência leitora, embora se reconheçam outros obstáculos que decorrem desta perturbação. Os aspetos negativos são, aliás, os que se encontram de forma mais explícita nas investigações levadas a cabo nesta área. No entanto, sabemos que a dislexia é responsável pela promoção de um pensamento divergente, que potencia capacidades suplementares face aos seus pares sem dislexia, trazendo múltiplos benefícios à vida diária. $\mathrm{O}$ estudo procurou conhecer as conceções de adultos com dislexia sobre os fatores positivos que Ihe estão associados, tendo-se seguido, para o efeito, um paradigma qualitativo e um estudo de tipo exploratório-descritivo. Os participantes, 17 adultos com diagnóstico de dislexia, de várias idades e distintos percursos pessoais, académicos e laborais, responderam a um inquérito por questionário, com questões essencialmente abertas. Os textos recolhidos através desse instrumento foram dissecados pela técnica de análise de conteúdo, focando-se o presente artigo na quarta categoria de análise e respetivas subcategorias (Aspetos positivos associados à Dislexia - Estratégias de Superação; Vantagens). Os resultados obtidos reiteram a perspetiva identificada na literatura, uma vez que, para estas pessoas, a dislexia se tornou, maioritariamente, uma característica com consequências nefastas. Ainda assim, os participantes consideram que a dificuldade thes permitiu desenvolver capacidades importantes que, de outra forma, não teriam alcançado. São pessoas fortemente determinadas e motivadas, que se focam na procura das melhores soluções para alcançar os resultados pretendidos, através de estratégias para o controlo da ansiedade e manutenção da atenção/concentração nas tarefas, fatores úteis a um desempenho académico ou profissional de qualidade.

Palavras-chave: Dificuldades de Aprendizagem Específicas; Dislexia; Adultos; Aspetos Positivos.

\section{Is Dyslexia an Advantage? A Different Look at the Difference}

Abstract: Dyslexia is a Specific Learning Difficulty associated mainly with weaknesses in reading skills, although other obstacles are recognized as arising from this disorder. The negative aspects are, in fact, those most explicitly found in the research carried out in this area. However, we know that dyslexia is responsible for promoting divergent thinking, which enhances additional abilities compared to non-dyslexic peers, bringing multiple benefits to daily life. The study sought to understand the conceptions of adults with dyslexia about the positive factors associated with it. For this purpose a qualitative and an exploratory-descriptive study was followed. The participants, 17 adults diagnosed with dyslexia, of various ages and different personal, academic and employment backgrounds, responded to a questionnaire survey, essentially with open questions. The texts collected through this instrument were dissected using the content analysis technique, focusing this article on the fourth category of analysis and its subcategories (Positive aspects associated with dyslexia - Overcoming strategies; Advantages). The results obtained reiterate the perspective identified in the literature, since, dyslexia has mostly become a characteristic with harmful consequences for these people. Nevertheless, the participants consider that the difficulty enabled them to develop important skills that they would not otherwise have achieved. They are strongly determined and motivated people, who focus on finding the best solutions to achieve the desired results, through strategies for controlling anxiety and maintaining attention/concentration on tasks, useful factors for quality academic or professional performance.

Keywords: Specific Learning Difficulties; Dyslexia; Adults; Positive Aspects. 


\section{Introdução}

As Dificuldades de Aprendizagem Específicas (DAE) ou, como apelidadas pela American Psychiatric Association (2014), Perturbações da Aprendizagem Específica, englobam transtornos na área da leitura (precisão, ritmo ou fluência e compreensão da leitura), da escrita (precisão ortográfica, gramatical e da pontuação e clareza ou organização da expressão escrita) e do cálculo (sentido numérico, memorização de factos aritméticos, cálculo preciso ou fluente e raciocínio matemático preciso). Estas têm sido alvo de investigação e publicação à escala internacional, procurando-se aprofundar 0 conhecimento das suas características, para criação de diferentes formas de as avaliar e de intervir psicopedagogicamente e com eficácia. Denota-se, no entanto, que o foco dos trabalhos desenvolvidos se centra, maioritariamente, em crianças e jovens que se encontram no sistema educativo.

No que diz respeito, especificamente, à dificuldade/perturbação na área da leitura (dislexia), temática central deste artigo, sabe-se, no entanto, que "(...) no es una dificultad transitoria en la persona que la padece, sino que persiste en la vida adulta." (Jiménez, Gregg \& Díaz, 2004, p. 64), momento em que estes indivíduos perdem a égide associada ao contexto escolar, ganhando, por outro lado, um conhecimento e uma experiência de vida que thes permite refletir criticamente acerca do seu percurso e das dificuldades a ele associadas. Neste sentido, importa escutar estas pessoas, acreditando-se que podem contribuir para um conhecimento mais aprofundado desta característica individual, tão inesperada face às suas capacidades cognitivas.

Foi neste contexto que se desenvolveu uma investigação qualitativa, com base em narrativas de adultos com dislexia, procurando-se responder à seguinte questão de partida: De que forma é que adultos com dislexia caracterizam esta dificuldade de aprendizagem específica?

Com base nesta questão foram delineados os seguintes objetivos:

1. Conhecer as conceções de adultos com dislexia sobre as dificuldades encontradas ao longo da sua vida;

2. Conhecer as conceções de adultos com dislexia sobre os fatores positivos que associam ao facto de terem esta DAE;

3. Identificar a articulação existente entre a opinião dos participantes do estudo;

4. Confrontar os pareceres de adultos com dislexia com a literatura científica.

Apesar de a dislexia estar associada, maioritariamente, a aspetos negativos, o que transparece, desde logo, pelo facto de ser considerada uma dificuldade de aprendizagem, os objetivos foram traçados para ir além desta realidade, procurando-se encontrar outros fatores, não só positivos como, eventualmente, vantajosos para os indivíduos com dislexia. Acredita-se que a capacidade reflexiva dos adultos, com um maior grau de distanciamento face aos momentos pré e pós diagnóstico, frequentemente instáveis e conturbados, permite alcançar esta premissa que, de certa forma, se constitui como um fator inovador face à maioria dos estudos já desenvolvidos. Neste sentido, ainda que a investigação tenha cumprido todos os objetivos traçados, o artigo que aqui se apresenta, centrar-se-á no segundo objetivo elencado.

A investigação iniciou-se por uma pesquisa bibliográfica, que enquadrou teoricamente a temática em estudo, cujas principais ilações se encontram no ponto seguinte deste texto. No ponto 3 apresenta-se a Metodologia, nomeadamente a tipologia de estudo desenvolvido, os participantes, adultos com dislexia, que colaboraram com as suas perspetivas com base nas vivências passadas, a técnica, instrumento e procedimentos adotados e a respetiva técnica de análise de dados. A partir da análise de conteúdo efetuada sobre os dados recolhidos no estudo, foi possível identificar resultados pertinentes, que serão explanados na quarta secção do texto, a que se seguirá uma síntese das conclusões obtidas. 


\section{Enquadramento Teórico}

A dislexia tem sido caracterizada por inúmeros autores, sendo algumas definições bastante controversas e, até, contraditórias. A definição apresentada em 2002 pela Associação Internacional de Dislexia parece ser a que continua, passados quase vinte anos, a reunir maior consenso na comunidade científica. Na página web desta Associação é possível ler o seguinte:

Dyslexia is characterized by difficulties with accurate and / or fluent word recognition and by poor spelling and decoding abilities. These difficulties typically result from a deficit in the phonological component of language that is often unexpected in relation to other cognitive abilities and the provision of effective classroom instruction. Secondary consequences may include problems in reading comprehension and reduced reading experience that can impede growth of vocabulary and background knowledge (International Dyslexia Association, 2002, s.p.).

Com base nesta definição e em articulação com a perspetiva de diversos autores, nacionais (Moura; Pereira \& Simões, 2018; Serra \& Estrela, 2007) e internacionais (Lyon, Shaywitz, \& Shaywitz, 2003; Montarnal, 2012; Shaywitz, 2020; Snowling, Hulme \& Nation, 2020) é possível caracterizar a dislexia como uma dificuldade com origem neurobiológica, que acarreta uma fraca precisão, ritmo e/ou fluência no reconhecimento de palavras, o que se traduz numa fraca capacidade de compreensão leitora e noutras áreas, nomeadamente na escrita. Segundo os autores, esta dificuldade é inesperada em relação a outras competências/habilidades cognitivas, não sendo sinal de um baixo QI mas sim de uma fraca capacidade de concentração/atenção nas tarefas e de gestão do tempo/espaço, o que gera consequências secundárias, ao nível académico, pessoal/emocional, social, entre outras.

Analisando estas características, percebe-se que são maioritariamente negativas, o que não invalida que à dislexia estejam associados aspetos que podem, de forma direta ou indireta, trazer benefícios aos sujeitos que recebem este diagnóstico. Apesar de ainda escassas, é possível identificar algumas publicações que se dedicam à exploração deste lado mais profícuo da dislexia, o que se observa, desde logo, pelo título de algumas delas como 'The Gift of Dyslexia: Why Some of the Smartest People Can't Read...and How They Can Learn' de Ronald David e Eldon Braun ou 'The Dyslexic Advantage: Unlocking the Hidden Potential of the Dyslexic Brain' de Brock Eide e Fernette Eide.

Os benefícios identificados na literatura, apontam, segundo Schneps (2014), para uma maior capacidade dos sujeitos com dislexia analisarem o panorama geral dos acontecimentos, tanto do ponto de vista literal como figurativo, reconhecendo elementos de um ambiente ou cenário (ex. discriminar figuras que representam situações impossíveis) que passam despercebidos a outros sujeitos. O mesmo autor explica que esta realidade se deve ao facto da dislexia acarretar fragilidades ao nível da atenção e processamento visual, imprescindíveis para uma leitura precisa, aumentando, no entanto, a sua consciência/perceção global.

Estas diferenças na forma como se perceciona e processa a informação podem influenciar negativamente a capacidade leitora, mas contribuem positivamente para outras habilidades, já que permitem pensar fora da caixa. A Associação britânica Made by Dyslexia (2020; s.d.) identifica, a este nível, seis áreas a que chama de Dyslexic Thinking Skills:

i) Visualização (movimentar, fazer, inventar) - capacidade de interagir e interpretar objetos, espaços, ideias corpóreas e novos conceitos;

ii) Imaginação (criar, interpretar) - capacidade para criar trabalhos originais ou para pensar criativamente sobre uma ideia;

iii) Comunicação (explicar, narrar histórias) - capacidade para transmitir uma mensagem de forma clara e envolvente;

iv) Raciocínio (simplificar, analisar, decidir, visionar) - capacidade para compreender padrões, analisar hipóteses e tomar decisões lógicas; 
v) Conexão (compreender-se, compreender os outros, influenciar, criar empatia) capacidade para se conectar consigo (autoconhecimento) e com os outros, gerando empatia e habilidade para influenciar outras pessoas;

vi) Curiosidade (aprender, aprofundar, ter energia, fazer) - capacidade e motivação para explorar ideias, de forma constante e dinâmica.

Este pensamento distintivo e inovador permite que as pessoas com dislexia se tornem uma mais valia para múltiplas tarefas, sendo reconhecido o seu potencial para 0 desenvolvimento de novas ideias e o consequente crescimento das organizações e, de uma forma geral, da sociedade. "After all, our conceptions of "advantage" and "disadvantage" have meaning only in the context of the task that needs to be performed. [...] Impairments in one area can lead to advantages in others, and it is these differences that drive progress." (Schneps, 2014, p. 25).

\section{Metodologia}

Como referido previamente, a investigação alicerçou-se no levantamento bibliográfico que identificou uma parca produção científica que, por um lado, identifique o flanco positivo da dislexia e, por outro, recolha dados junto de sujeitos disléxicos, com mais de 18 anos, e não apenas junto de crianças/jovens com dislexia. Esta constatação sustentou a delimitação da temática do estudo, bem como a formulação da questão de investigação e dos objetivos, identificados na introdução deste artigo, que se foca no objetivo 2, procurando-se identificar e descrever as perceções de adultos com diagnóstico de dislexia, acerca dos fatores positivos que associam ao facto de terem esta DAE.

Reconhece-se que a seleção das especificidades metodológicas deve ser estruturada em torno da problemática da investigação e dos objetivos visados, mas também dos atores intervenientes no processo, pelo que se seguiu um paradigma qualitativo, por se considerar que importava recolher, em profundidade, a perspetiva dos que vivenciam experiências quotidianas tendo dislexia. As investigações qualitativas têm este grande intento de compreender a realidade social estudada, centrando-se na análise das vivências dos participantes, a partir de dados que traduzem as suas perspetivas (Clark \& Creswell, 2015; Fortin, 2006).

\subsection{Tipo de Estudo}

A investigação realizada enquadra-se numa tipologia exploratório-descritiva.

O caráter exploratório deve-se ao facto do estudo procurar desbravar uma temática em que o conhecimento ainda é pouco consistente e em que o problema não se encontra delimitado de forma pormenorizada, o que poderá trazer resultados que sirvam de base a outros estudos (Dias, 2010). Por outro lado, a pesquisa é descritiva porque visa clarificar as características de um acontecimento ou de um grupo de pessoas, neste caso adultos com dislexia, a partir de um conhecimento teórico mais sustentado, que serve de alicerce para o estabelecimento de relações entre variáveis, numa dualidade entre a teoria e a prática/realidade, refletindo sobre ela (Clark \& Creswell, 2015; Dias, 2010).

A articulação da vertente descritiva com a exploratória é particularmente usada por investigadores sociais preocupados com situações reais, tendo em conta que regista, analisa e interpreta factos, identificando os elementos que contribuem para que determinado fenómeno ocorra, o que gera formulação de ideias e hipóteses de dedução lógica (Marconi \& Lakatos, 2017). 


\subsection{Participantes}

Os participantes que integraram a investigação foram selecionados em função da natureza do estudo e, por essa razão, constituiu-se uma amostra não probabilística, intencional e por conveniência, pautada por critérios pré-definidos (Almeida \& Freire, 2017; Carmo \& Ferreira, 2015; Coutinho, 2013).

A investigadora procedeu a um contacto direto com pessoas adultas (com mais de 18 anos), portuguesas, com diagnóstico de dislexia, a que tinha acesso direto, que, por sua vez, fizeram chegar o instrumento de recolha de dados a outros sujeitos com iguais características, num procedimento tipo bola de neve ou amostragem em cadeira (Clark \& Creswell, 2015; Mertens, 2020). Com base nesta técnica, foi possível integrar no estudo 17 participantes, 12 do género feminino e 5 do masculino, com idades compreendidas entre os 20 e os 52 anos, a que se associou uma letra do alfabeto ( $A$ a $Q$ ), no sentido de respeitar o anonimato dos sujeitos.

Tabela 1. Idade dos participantes.

\begin{tabular}{lc}
\hline \multicolumn{1}{c}{ Idades } & Número absoluto \\
\hline 18-27 anos & 8 \\
28-37 anos & 2 \\
38-47 anos & 4 \\
48-57 anos & 3 \\
58-67 anos & 0 \\
Mais de 67 anos & 0 \\
\hline
\end{tabular}

A partir dos dados recolhidos foi, ainda, possível perceber, a formação e profissão dos participantes, o que pode, de certa forma, contribuir para complementar a resposta ao objetivo em foco nesta investigação, já que evidencia que, apesar de a dislexia poder trazer dificuldades aos sujeitos, não os impede de seguir estudos superiores e de exercer profissões com elevado nível de exigência e responsabilidade.

Considerando, oito níveis de habilitações literárias, verificou-se a seguinte distribuição: $1 .^{\circ}$ Ciclo do Ensino Básico (CEB) - 0; 2. CEB - 1; 3. CEB - 1; Ensino Secundário - 4; Curso de Ensino Pós-secundário Não Superior (CET) ou Curso Técnico Superior Profissional $(C T e S P)$ - 4; Licenciatura - 3; Mestrado - 4 e Doutoramento - 0.

As atividades profissionais são bastante díspares, indo desde um operário da construção civil, funcionário de armazém ou costureira, a estudantes do Ensino Superior, educadores de infância, professores e designers.

\subsection{Instrumentos, Técnicas e Procedimentos de Recolha de Dados}

Sendo o número de participantes reduzido, poder-se-ia perpetivar a recolha de dados através de diversos instrumentos, sendo o mais comum neste tipo de situações o inquérito por entrevista. No entanto, considerou-se, com base nas perspetivas de Gil (2019) e Marconi e Lakatos (2017), que a adotação deste tipo de instrumento poderia, de alguma forma, exercer uma influência, consciente ou inconsciente, sobre as respostas dos inquiridos, nomeadamente pelo constrangimento que a exposição de um assunto sensível, como é o caso da dislexia, pode gerar nos inquiridos. Para além disso, o facto de alguns participantes serem conhecidos da investigadora, poderia gerar algum receio, nomeadamente no que diz respeito à revelação da identidade, o que aumentaria o risco de obter respostas falseadas ou a ocultação de dados importantes. Por outro lado, e ainda segundo os mesmos autores, os inquéritos por questionário garantem o anonimato dos intervenientes, permitindo-Ihes uma maior flexibilidade de respostas, evitando uma influência direta do investigador. 
Pelas razões expostas, optou-se por construir um questionário, tendo por base a questão de investigação e os objetivos, tal como defendem Prodanov e Freitas (2013), Fortin (2003), entre muitos outros autores de referência na área da metodologia de investigação. Este traçou-se a partir dos pressupostos da investigação em ciências sociais e humanas, coadjuvada pela revisão da literatura sobre o tema em estudo e incluiu essencialmente duas partes, uma inicial de caracterização dos respondentes e uma segunda com questões abertas que procuravam, num cômputo geral, recolher a perceção dos participantes sobre a forma como estes encaram a dislexia enquanto característica que os acompanhou ao longo da vida. A opção por este tipo de questões prende-se com a necessidade de não condicionar a liberdade nas respostas dadas, evitando condicionamentos gerados a partir de opções pré-determinadas.

O procedimento para a operacionalização da aplicação dos questionários foi estruturado em vários momentos. Começou-se por construir o consentimento informado, livre e esclarecido e o instrumento (questionário), que foi depois validado por consulta de especialistas e realização de pré-teste. Após alguns ajustes o instrumento foi enviado por email, com base na autorização prévia dos primeiros participantes, que enviaram depois o consentimento/questionário a outros adultos com dislexia, com base nos seus contactos pessoais. As respostas foram recolhidas seguindo-a a mesma lógica. Com base nos dados recolhidos, seguiu-se a técnica de análise de conteúdo.

\subsection{Técnica de Análise de Dados}

Nas investigações qualitativas recolhem-se, segundo Clara Coutinho (2013), dados que são, geralmente, de índole mais aberto e flexível, obtendo-se uma vasta quantidade de informação descritiva, a qual implica organização, simplificação e agrupamento, ou seja, um processo de análise de conteúdo. Esta permite interpretar, da forma mais aprofundada possível, o fenómeno em estudo, dando lugar a inferências que perspetivam regularidades e similaridades dos dados, destacando a pertinência, conformidade, objetividade e fidelidade do seu conteúdo.

A análise de conteúdo foi, portanto, a técnica de análise de dados aplicada, tendo sido adotado um conjunto de procedimentos sistemáticos, que se podem estruturar, segundo Bardin (2009), em três fases: (1) Pré-análise, que inclui a recolha e preparação do material, em função de regras definidas pelos investigadores; (2) Exploração do material, onde são aplicadas as regras de ação anteriormente estipuladas e que permitem a codificação e categorização de acordo com a significância e (3) Tratamento dos dados, que se traduz na interpretação, dedução e síntese dos resultados.

A investigação desenvolvida seguiu estas fases, que permitiram codificar os dados recolhidos, em função dos objetivos traçados, através da identificação de categorias e subcategorias, à priori, a partir do quadro teórico e, outras, que surgiram à posteriori, com base na análise exploratória dos dados. As categorias, subcategorias e os respetivos indicadores, encontram-se expressos na Tabela 2.

Tabela 2. Categorias, subcategorias e indicadores adotados na análise de conteúdo

\begin{tabular}{lll}
\hline \multicolumn{1}{c}{ Categorias } & Subcategorias & \multicolumn{1}{c}{ Indicadores } \\
\hline $\begin{array}{l}\text { Diagnóstico da } \\
\text { dislexia }\end{array}$ & Processo & $\begin{array}{l}\text { Menções acerca dos procedimentos adotados } \\
\text { para obtenção do diagnóstico, nomeadamente as } \\
\text { fases e pessoas envolvidas } \\
\text { Menções que remetam para o momento do } \\
\text { diagnóstico (precoce/tardio) }\end{array}$ \\
$\begin{array}{l}\text { Dificuldades/ } \\
\begin{array}{l}\text { Aspetos negativos } \\
\text { associados à } \\
\text { dislexia }\end{array}\end{array}$ & Leitura e & $\begin{array}{l}\text { Menções que destaquem dificuldades na leitura } \\
\text { (decifração ou compreensão) e na escrita }\end{array}$ \\
& $\begin{array}{l}\text { Escrita } \\
\text { Concentração/ }\end{array}$ & $\begin{array}{l}\text { Menções a dificuldades de atençãa ou ou competência textual) } \\
\text { concentração }\end{array}$
\end{tabular}


Vol. 9 | Investigação Qualitativa em Ciências Sociais: Avanços e Desafios

\begin{tabular}{|c|c|c|}
\hline Categorias & Subcategorias & Indicadores \\
\hline \multirow{5}{*}{$\begin{array}{l}\text { Perceção/Reação } \\
\text { de outras pessoas } \\
\text { face à dislexia }\end{array}$} & $\begin{array}{l}\text { Gestão do } \\
\text { tempo e do } \\
\text { espaço }\end{array}$ & $\begin{array}{l}\text { Menções à fraca autonomia na gestão individual } \\
\text { do tempo e organização espacial }\end{array}$ \\
\hline & $\begin{array}{l}\text { Emocionais/Rel } \\
\text { acionamento }\end{array}$ & $\begin{array}{l}\text { Menções a fragilidades emocionais ou de } \\
\text { relacionamentos com os outros }\end{array}$ \\
\hline & $\begin{array}{l}\text { Professores e } \\
\text { técnicos }\end{array}$ & $\begin{array}{l}\text { Menções à forma como os profissionais } \\
\text { reagiram perante a dislexia }\end{array}$ \\
\hline & Familiares & $\begin{array}{l}\text { Menções à forma como a família reagiu perante } \\
\text { a dislexia }\end{array}$ \\
\hline & Pares & $\begin{array}{l}\text { Menções à forma como os colegas/amigos } \\
\text { (pares) reagiram perante a dislexia }\end{array}$ \\
\hline \multirow{2}{*}{$\begin{array}{l}\text { Aspetos positivos } \\
\text { associados à } \\
\text { dislexia }\end{array}$} & $\begin{array}{l}\text { Estratégias de } \\
\text { superação }\end{array}$ & $\begin{array}{l}\text { Menções a estratégias que o sujeito disléxico } \\
\text { tenha adotado para superar eventuais } \\
\text { dificuldades }\end{array}$ \\
\hline & Vantagens & $\begin{array}{l}\text { Menções a fatores que o sujeito disléxico } \\
\text { entenda como vantagens face a pessoa não } \\
\text { disléxicas }\end{array}$ \\
\hline
\end{tabular}

Para organizar os dados em função da categorização apresentada na tabela, recorreu-se ao software WebQDA, tendo em conta que este auxilia a disposição dos dados em categorias, através de processos de codificação, controlo, filtragem, pesquisa e questionamento, possíveis pela facilidade de edição, visualização e interligação de ficheiros que o software apresenta (Souza, Costa \& Moreira, 2011).

No âmbito do presente artigo, os dados apresentados e a discussão dos resultados centra-se na última categoria da Tabela 2 (aspetos positivos associados à dislexia) e nas respetivas subcategorias, tendo-se selecionado excertos dos discursos dos participantes. Os fragmentos de texto procuraram ir ao encontro dos indicadores definidos e respeitar, tanto quanto possível, o sentido das narrativas integrais, já que, tal como Bogdan e Biklen (2013, p. 252), se acredita que "um bom trabalho qualitativo é documentado com boas descrições provenientes dos dados para ilustrar e substanciar as asserções feitas".

\section{Apresentação dos Dados e Discussão dos Resultados}

Antes de se passar à apresentação dos dados recolhidos e à discussão dos resultados obtidos a partir da análise dos elementos da categoria 'Aspetos positivos associados à dislexia', foco deste trabalho, importa destacar três aspetos a partir dos quais é possível realizar algumas deduções lógicas.

O primeiro diz respeito ao facto de terem sido contactados vários adultos com dislexia que não desejaram participar na investigação ou, mostrando-se inicialmente disponíveis, acabaram por não responder ao questionário. Esta situação pode ser um indicador do receio de exposição ou da dificuldade em responder às questões colocadas, o que faz com que se possa inferir que, por um lado, a dislexia se tornou um grande fardo na vida destas pessoas, fazendo com que não desejem relembrar situações e momentos penosos e que, por outro, estas poderão ter a tendência para esconder o seu diagnóstico aos elementos da comunidade que com elas convivem, o que pode intensificar as dificuldades sentidas pela falta de apoio e compreensão por parte dos seus pares.

O segundo aspeto a destacar prende-se com o facto de, dos 17 participantes, 4 não terem incluído, na resposta ao questionário, elementos positivos associados à dislexia. As suas respostas basearam-se, essencialmente, nas dificuldades e problemas que esta DAE acarretou para os seus percursos de vida. 
A terceira nota está associada à discrepância da densidade textual entre as categorias em que a análise de conteúdo foi organizada. Verificou-se que, mesmo quando os inquiridos incluíram, nas suas respostas, dados relevantes para os vários blocos temáticos, a categoria 'Aspetos positivos associados à dislexia' é sempre a mais pobre em termos de quantidade de texto, centrando-se as respostas nas dificuldades encontradas ao longo da vida.

Pelos fatores apresentados, depreende-se que, embora a dislexia tenha uma influência positiva na vida dos sujeitos, está bastante mais associada a circunstâncias pungentes, com implicações nas várias fases do desenvolvimento humano. Estas dificuldades, ainda que sejam uma realidade, geram também uma atitude de perseverança e determinação na pessoa com dislexia, implicando esforço individual para ultrapassar os vários desafios encontrados no dia a dia, o que Dweck (2018) apelida de mindset. Efetivamente, os participantes no estudo reconhecem que são pessoas mais resilientes ("lutadores" - H; N T) e que apresentam particularidades vantajosas face aos seus pares - "a dislexia [...] torna-nos pessoas únicas e especiais." (C), acreditando que é possível ter muito sucesso, escolar, profissional e pessoal/social, enquanto resultado de uma forma diferente de estar na vida: "Hoje a dislexia é para mim uma bandeira que hasteio com orgulho, pois quero mostrar ao mundo que é possível conviver com a dislexia de forma natural, vencer os medos e limitações que ela impõe e ter uma vida normal." (C); "Posso dizer que ser dislexia me faz crescer todos os dias como pessoa e profissional." (M).

Para que a dislexia possa ser considerada uma característica positiva, os adultos aplicaram, ao longo do seu percurso de vida, estratégias para enfrentarem os obstáculos com que se iam confrontando, a que chamam "truques" $(A)$, "truques, técnicas" $(\mathrm{O})$, "estratégias de correção consciente" $(F)$, tais como:

- Aceitar a dislexia - "fui percebendo que tinha de aprender a viver com a dislexia de forma natural, aceitá-la e fazer dela motor de arranque para ultrapassar todas as barreiras" (C);

- Compreender que a dislexia não é sinónimo de fraca inteligência - "A sociedade devia olhar com outros olhos para os disléxicos porque eles ficam ao nível dos melhores, a perceção do conhecimento é diferente, mas acontece." (K), "Uma pessoa disléxica não quer dizer que não seja inteligente e saudável" (E);

- Manter a motivação - "tenho uma motivação enorme que é contrariar quem me diz "não és capaz". Aí é certo que tenho sempre motivação para não desistir e mostrar que consigo" (K); "(...) não é uma limitação, antes pelo contrário é um motivo para continuar a lutar procurando sempre ser melhor em tudo quanto faço" (C);

- Controlar a ansiedade - "tento estar calma, para não atrapalhar a vida escolar e a vida profissional" (D);

- Apostar em mecanismos para aumentar a atenção/concentração - "O que funcionava mesmo era estar atento, cumprir com o que me pediam, nunca ficar ao meio na planta da sala e trabalhar muito" (K);

- Praticar e estudar de forma intensiva - "Há que ultrapassar as dificuldades, trabalhar, trabalhar, não há outra hipótese. Sei que trabalhava muito mais que os meus colegas. [...] Com trabalho todo o disléxico vence." (G), "muitas noites a estudar, muitos fins de semana sem ir à rua" $(\mathrm{H})$, "muita prática e exercícios especiais/específicos" $(\mathrm{J})$, "Fiz muitos exercícios e melhorei muito" (K);

- Desenvolver o gosto por áreas mais fragilizadas - "desenvolver algum gosto pela leitura e pela escrita" (C); "ler um parágrafo duas ou três vezes, até perceber a ideia" (K);

Para além destes comportamentos individuais, foi também possível verificar que os sujeitos com dislexia reconhecem como fatores positivos para o seu desempenho o acompanhamento de profissionais/técnicos - "a dislexia é vista como uma conquista de cada dia [...] procuro combater e tentar ser melhor, pois não é nenhum percalço, nem pedra no sapato como já me referiram, mas sim um obstáculo a ultrapassar cada dia [...] com o apoio de bons profissionais" (O), familiares - "Algumas vezes peço ao meu marido, para ler, eu fico a ouvir e assim interpreto melhor." (L) ou de pessoas do seu círculo de amizade - "peço sempre que me corrijam e que me expliquem a melhor maneira de o dizer ou escrever" (M). 
As estratégias descritas, permitiram que muitos dos sujeitos com dislexia, agora adultos, conseguissem vislumbrar a dislexia como uma característica que levou ao desenvolvimento de um conjunto de capacidades que, de outra forma, não apresentariam, indo ao encontro das perseptivas de diversos autores, nomeadamente de Schneps (2014) e da Associação Made by Dyslexia (2020; s.d).

\section{Conclusões}

O estudo qualitativo, de tipo exploratório-descritivo, permitiu reconhecer que a dislexia acarreta muitos obstáculos à vida dos sujeitos. No entanto, a maturidade associada à idade adulta, permite refletir sobre o percurso de vida e reconhecer que a DAE também aporta características únicas, que se traduzem em vantagens.

Ainda que não seja possível generalizar os resultados, face à dimensão da amostra, denota-se que os participantes com dislexia são sujeitos muito determinados e motivados, trabalhando de forma insistente para alcançar os resultados pretendidos, mesmo em áreas em que se sentem mais fragilizados. São, ainda, pessoas que desenvolvem estratégias para o controlo da sua ansiedade e manutenção da atenção/concentração nas tarefas, fatores úteis a um desempenho académico ou profissional de qualidade. A literatura aponta, por seu turno, que devido a esta forma diferente de organizar o pensamento, são pessoas que têm capacidades de visualização, imaginação, comunicação, raciocínio lógico, conexão, consigo e com os outros, e curiosidade.

Perante as conclusões, constatasse que a voz dos adultos com dislexia pode contribuir para conhecer em profundidade esta dificuldade de aprendizagem, alargando o horizonte de perspetivas e valorizando formas distintas de pensar e agir perante o mundo que nos rodeia. Sugere-se, por isso, que possam ser desenvolvidos estudos futuros que vão ao encontro desta premissa, nomeadamente através do alargamento do número de participantes com dislexia, que possam expor, na primeira pessoa, as particularidades que os caracterizam.

\section{Referências}

Almeida, L., \& Freire, T. (2017). Metodologia de Investigação em Psicologia e Educação. Psiquilibrios.

American Psychiatric Association (2014). Manual de Diagnóstico e Estatística das Perturbações Mentais - DSM V. Climepsi Editores

Bardin, L. (2009). Análise de conteúdo. Edições 70.

Bogdan, R. \& Biklen. S. (2013). Investigação qualitativa em educação: uma introdução à teoria e aos métodos. Porto Editora

Carmo, H. \& Ferreira, M. (2015). Metodologia da investigação: guia para auto-aprendizagem Universidade Aberta

Clark, V.L. \& Creswell, J. W. (2015). Understanding Research: A consumer's guide. Pearson Education

Coutinho, C. (2013). Metodologia de Investigação em Ciências Sociais e Humanas: Teoria e Prática. Almedina.

Dias, M. (2010). Planos de Investigação. Avançando Passo a Passo. Rainho \& Neves

Dweck, C. (2018). Mindset, a atitude mental para o sucesso. Vogais \& Companhia

Fortin, M.-F. (2003). O Processo de investigação: Da concepção à realização. Lusociência.

Fortin, M.F. (2006). Fundamento e etapas do processo de investigação. Lusodidacta. 
Gil, A. (2019). Métodos e técnicas de pesquisa social. Editora Atlas

International Dyslexia Association (2002). Definition Consensus Project. Disponível em: https://dyslexiaida.org/definition-consensus-project/

Lei n. ${ }^{85 / 2009}$ de 27 de agosto da Assembleia da República (2009). Diário da República: 1. a série, $\mathrm{N}$. ㅇ 166. https://dre.pt/pesquisa/-/search/488826/details/maximized

Lyon, R., Shaywitz, S., \& Shaywitz, B. (2003). A Definition of Dyslexia. Annals of Dyslexia, 53, pp. 1-14.

Made by Dyslexia (2020). Connect the Spots 2020. Disponível em: https://www.madebydyslexia.org/assets/downloads/ConnectTheSpotsReport.pdf

Made by Dyslexia (s.d.). Spelling it out. Disponível em: https://www.madebydyslexia.org/assets/downloads/spelling-it-out.pdf

Marconi, M. \& Lakatos, E. (2017). Fundamentos de metodologia científica. Atlas

Mertens, D. (2020). Research and Evaluation in Education and Psychology: Integrating Diversity With Quantitative, Qualitative, and Mixed Methods. Sage

Montarnal, A. (2012). Adultes dyslexiques: concrètement que faire?. Éditions Tom Pousse

Moura, O.; Pereira, M.; Simões, M. (coord.). (2018). Dislexia - Teoria, Avaliação e Intervenção. Pactor.

Prodanov, C. \& Freitas, E. (2013). Metodologia do trabalho científico: métodos e técnicas da pesquisa e do trabalho académico. Universidade Feevale.

Schneps, M. H. (2014). The Advantages of Dyslexia. Scientific American Mind, 26(1), 24-25.

Serra, H., \& Estrela, M. F. (2007). Dislexia e Perturbações Associadas: Memória e Atenção. Cadernos de Estudo, 5, 93-115.

Shaywitz, S. \& Shaywitz, J. (2020). Overcoming Dyslexia: Completely Revised and Updated. Random House USA Inc

Snowling, M.; Hulme, C. \& Nation, K. (2020). Definine and understanding dyslexia; past, present and future. Oxford Review of Education, 46(4), 501-513 